\title{
Patrones de distribución de insectos de escama sobre cuatro especies del arbolado urbano de Bogotá, Colombia
}

\author{
Distribution patterns of scale insects \\ on four species of the tree-lined urban \\ of Bogotá, Colombia
}

\section{Padrões de distribuição de insetos de escama sobre quatro espécies de arborização urbana de Bogotá, Colômbia}

\author{
1Raúl Hernando Posada Almanza \\ ${ }^{1}$ Biólogo, Magister en Microbiología, Doctor en Ciencias - Ecología \\ Dirección de Investigación. Zenkinoko SAS, Diagonal 151b 136A-75 Cs93. - Bogotá, Colombia. \\ ${ }^{1}$ raulposada@hotmail.com
}

\section{Resumen}

Ciertas grandes ciudades llevan a cabo programas de aforestación en un intento de solucionar problemas relacionados con la polución. Esta incorporación de árboles y arbustos ha abierto las puertas a la incorporación de insectos de escama con patrones de distribución y densidad que dependen de múltiples factores. Esta investigación evaluó los patrones de distribución de insectos de escama sobre cuatro especies arbóreas usadas en la aforestación de la zona urbana de Bogotá, Colombia. La presencia y densidad de insectos de escama en árboles georeferenciados fue valorada y variados análisis geoestadísticos fueron realizados para describir su presencia y densidad como función de la distancia entre árboles. En general, los insectos de escama estuvieron distribuidos en áreas con altas densidades. Una distribución no azarosa fue evidente a una escala más detallada, con insectos presentes en pequeños grupos. Sin embargo, se encontraron insectos de escama sobre Ficus soatensis que presentaron una distribución completamente azarosa. El estudio de los patrones de distribución de insectos de escama en condiciones urbanas, en conjunto con estudios complementarios, puede contribuir a entender su respuesta a ambientes multifactoriales y provee datos valiosos para usar en el manejo de plagas.

Palabras clave: Croton bogotensis, Eugenia myrtifolia, Ficus soatensis, plagas, Schinus molle, distribución espacial.

\section{Abstract \\ Certain large cities are carrying out programs of afforestation in an attempt to solve problems related to pollution. This incorporation of trees and shrubs has opened the doors to the incorporation of scale insects with patterns of distribution and density that is dependent on many factors. This research evalua- ted the distribution patterns of scale insects on four tree species used in afforestation in the urban area of Bogota, Colombia. The presence and density of scale insects in trees georeferenced was valued and varied geostatistical analysis were made to describe its presence and density as a function of the distan- ce between trees. In general, the scale insects were distributed in areas with high densities. A distribution}


not random was evident at a more detailed scale, with insects present in small groups. However, were no scale insects on Ficus soatensis that presented a completely random distribution. The study of the patterns of distribution of scale insects in urban conditions, in conjunction with complementary studies, can contribute to understand your reply to environments multifactorial and provides valuable data for use in the management of pests.

Key-words: Croton bogotensis, Eugenia myrtifolia, Ficus soatensis, pest, Schinus molle, spatial distribution.

\section{Resumo}

Algumas grandes cidades organizam programas de arborização com a finalidade de resolver problemas relacionados com a poluição. Essa incorporação da árvores e arbustos tem abrido as portas a chegada de insetos escama com padrões de distribuição e densidade que depende de múltiplos fatores. Este estudo avaliou padrões de distribuição de insetos de escama sobre quatro espécies arbóreas usadas na arborização de Bogotá, Colômbia. A presença e densidade de insetos de escama foram distribuídas em áreas de altas densidades. Uma distribuição que não foi ao acaso foi evidente em escala mais detalhada, com insetos presente em grupos pequenos. No entanto, encontraram-se insetos de escama sobre Ficus soatensis que apresentaram uma distribuição completamente ao acaso. O estudo de padrões de distribuição de insetos de escama em condições urbanas, em conjunto com estudos complementários, pode ajudar a entender sua resposta a ambientes multifatoriais e prover dados valiosos para usar no manejo de pragas.

Palavras-chave: Croton bogotensis, Eugenia myrtifolia, Ficus soatensis, Pragas, Schinus molle, Distribuição espacial.

\section{Introducción}

El triángulo de enfermedades en plantas constituye uno de los paradigmas en la patología vegetal; por ejemplo, la presencia de una enfermedad causada por un agente biológico requiere de la interacción de un hospedero susceptible, un patógeno virulento y un ambiente favorable para el desarrollo de la enfermedad (Francl, 2001). Estos factores convergen en ciudades como Bogotá, que llevan a cabo programas de aforestación, donde la incorporación de plantas, estresores ambientales (Agrios, 2005; Dhingra \& Sinclair, 1995) y la abundancia de plagas potenciales con baja especificidad de hospedero (Marcano, Nienstaedt, Longa \& Malpica, 2006) proveen las condiciones apropiadas para la expresión de enfermedades. De otra parte, pero estrechamente relacionado, muchos insectos de escama tales como escamas de cera, escamas de tortuga, o cochinillas, son importantes como pestes de cosechas agrícolas que pueden dañar o matar las plantas por la reducción de los contenidos de sabia, la inyección de toxinas, transmitiendo virus (Fuchs, MarsellaHerrick, Loeb, Martinson \& Hoch, 2009; Martelli \&
Candresse, 2010; Retuerto, Fernandez-Lema, Rodríguez, \& Obeso, 2004; Vu, Eastwood, Nguyen \& Pham, 2006) o excretando gotas de miel en la superficie foliar (Becerra, González, Herrera \& Miano, 2006; Lampson \& Morse, 1993) a las cuales se puede adherir material particulado, obstruyendo y reduciendo las capacidades fotosintéticas de muchas especies (Graz, Garnernt \& Johnson, 2003; RamosMontaño, 2012). Los insectos de escama ayudan a la dispersión de la fumagina y realzan los efectos adversos de éstas en las plantas, p.ej. árboles de guayaba o mango en Egipto (Abd-Rabou, Badary \& Ahmed, 2012), o Phyllica arborea, donde Coccus sp en asociación con la fumagina Seiridium phylicae, reducen significantemente la producción de frutos de Phyllica (Ryan, Ortmann \& Herian, 2014). En ambientes urbanos, las cochinillas producen efectos que son similares a aquellos observados en cultivos comerciales (Hugh \& Cowles, 2009) y pueden ser un problema considerable, p.ej., cuando funcionan como agentes retardantes del crecimiento (Speight, Hails, Gilbert \& Foggo, 1998). 
Los insectos de escama son pequeños insectos del orden Hemíptera, suborden Sternorrhyncha. Ellos comprenden la superfamilia Coccoidea, la cual fue previamente encontrada en el hoy obsoleto grupo llamado "Homóptera". Los Coccidae son la familia de insectos de escama pertenecientes a la superfamilia Coccoidea. Son comunmente conocidos como escamas suaves, escamas de cera o escamas de Tortuga, e incluye los géneros Ceroplastes, Saissetia, Pulvinaria y Toumeyella. Los Pseudococcidae son una familia de insectos de escama que también pertenecen a la superfamilia Coccoidea. Pseudococcus y Colombiacoccus son géneros de cochinillas sin armadura dentro de esta familia y son comúnmente conocidos como piojos harinosos (Zamora, Martínez, Guerrero, Fuentes-Guerra \& Hernández, 2015). En Bogotá, varias especies de árboles y arbustos han sido usados para aforestar la ciudad; estas especies son afectados por plagas no específicas, incluidas algunos insectos de escamas (Agrios, 2005; Vurro, Bonciani \& Vannacci, 2010). Estas especies de hospederos incluyen: Sangregado (Croton bogotensis) Cuatrec, el cual no ha sido previamente reportado como afectado por cochinillas o insectos de escama, y Eugenia (Eugenia myrtifolia) Salisb., la cual es conocida por ser afectada por Colombiacoccus sp., Pseudococcus sp. y Saissetia spp. (Mahecha Vega et al., 2010). También está incluido en el programa de aforestación de Bogotá el Caucho sabanero (Ficus soatensis) Dugand, una especie con alta tolerancia a la contaminación ambiental, pero con presencia reportada de Pseudococcus spp. (Posada \& Forigua Acosta, 2008), Pulvinaria psiidi Mask, Toumeyella sp. y Ceroplastes sp. (Mahecha Vega et al., 2010), también la planta medicinal denominada falso pimiento (Schinus molle) (Schmidt et al., 2009), la cual es afectada por C. cundinamarcensis Mosquera, C. bicolor Hempel, Saissetia coffeae (Walker) y S. oleae Bern (AvilaOlesen, 2007; Mahecha Vega et al., 2010).

Adicional a la presencia de todos los elementos del triángulo de enfermedades en vegetales, muchos factores ambientales convergen en ciudades como Bogotá: a presencia de múltiples hospederos potenciales, condiciones climáticas, calentamiento urbano, flujos de aire, contaminación, fluctuaciones diarias, entre otros (Hanks \& Denno, 1993; Marcano et al., 2006; Meineke, Dunn, Sexton, \& Frank, 2013; Posada \& Ramos-Montaño, 2012; Yingping, Xianquian, Jingping \& Min, 1995) los cuales pueden influir en la incidencia y severidad de plagas y enfermedades, los cuales también pueden contribuir a su dispersión y distribución. Trabajando con F. soatensis, C. bogotensis, E. myrtifolia, Sambucus peruviana y S. molle, Posada y Ramos-Montaño (2012) mostraron como la densidad de insectos de escama estuvo relacionada a la precipitación local promedio y describieron diferencias en los patrones de preferencia de hospederos. En el mencionado estudio, la incidencia de insectos de escama en $C$. bogotensis fue inferior en áreas con precipitaciones inferiores a $600 \mathrm{~mm} / a n ̃ o$, mientras en $F$. soatensis la incidencia fue inferior en áreas con precipitación entre 600800 mm/año; en C. bogotensis, no hubo un patrón claro de severidad de plagas y enfermedades relacionado con las lluvias, mientras en $F$. soatensis la más alta incidencia de cóccidos estuvo directamente relacionada a la severidad de infestación. Esto demuestra la imposibilidad de generalizar como la distribución de los insectos de escama puede estar asociada de una manera específica y particular solamente a las condiciones de lluvia y también resalta la necesidad de investigaciones adicionales. Otros reportes en cóccidos, proveídos por instituciones gubernamentales en Bogotá, están limitados a la descripción de la presencia, nuevas especies, mecanismos de control implementados y reportes anuales de actividades, mientras existe una ausencia de investigaciones enfocadas a la evaluación del efecto de factores ambientales en este grupo de insectos.

Uno de los factores que determinan la dispersión de los insectos es la proximidad a los focos (en este caso los árboles con alta abundancia de la plaga), tomando en cuenta la presencia de diferentes árboles hospederos con diferentes grados de afectación a través de la ciudad. Es necesario iniciar identificando cómo la distribución de los hospederos afectados se relaciona con los patrones de distribución de insectos de escama tanto para una simple especie arbórea como para sistemas complejos de especies arbóreas, con el objeto de diseñar planes 
de manejo de plagas apropiados o estrategias de aforestación. Dentro de un área de influencia, los insectos plaga pueden estar distribuidos homogéneamente -al azar- o presentarse de forma agrupada (Sharov \& Liebhold, 1998; Wells, 1991).

En la presente investigación, los patrones de distribución de la presencia y severidad de insectos de escama fueron evaluados en cuatro especies del arbolado de Bogotá para determinar si la presencia y severidad de estas plagas están en función de la distancia entre árboles y su distribución. Los datos generados pueden indicar la influencia de la distribución de árboles en la infestación por insectos de escama y determinar la distancia mínima requerida entre potenciales hospederos individuales (solos o en una mezcla de especies) para minimizar la presencia y densidad de insectos de escama y así mejorar los sistemas de aforestación y la eficiencia de los programas de manejo de plagas. Específicamente se plantearon dos hipótesis: 1) la presencia de insectos de escama está asociada en un patrón agrupado a los hospederos potenciales en Bogotá, y 2) las plantas con altas densidades de insectos escama estarán localizadas a distancias cortas de otros árboles altamente afectados, mientras aquellos con bajas densidades de infestación estarán distribuidas azarosamente a lo largo de la ciudad.

\section{Materiales y métodos}

\section{Selección de especies vegetales}

Esta investigación fue realizada en la ciudad de Bogotá, Colombia, la cual está rodeada por los municipios de Chía, Cota, Funza, Mosquera, Soacha, Cáqueza, Chipaque y La Calera (Figura 1). Situa-

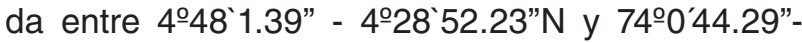
74-14 58.02" E; a $2640 \mathrm{msnm}$, con una temperatura promedio anual de $14^{\circ} \mathrm{C}$, y con dos periodos de lluvias separados por dos periodos secos y una población de aproximadamente 9,5 millones de habitantes. Para la selección de especies arbóreas, se filtró la lista de especies usadas para aforestar Bogotá, los mapas de su distribución en la ciudad y los registros de árboles con la más alta presencia de insectos de escama, las dos primeras fuentes fueron proveídas por la subdirección técnica y la última por la subdirección científica del Jardín Botánico de Bogotá José Celestino Mutis (JBJCM). Fueron seleccionados $C$. bogotensis, E. myrtifolia, $F$. soatensis y $S$. molle como especies arbóreas más importantes para evaluación, dada su abundancia, amplia distribución en la ciudad y la alta presencia registrada de insectos de escama.

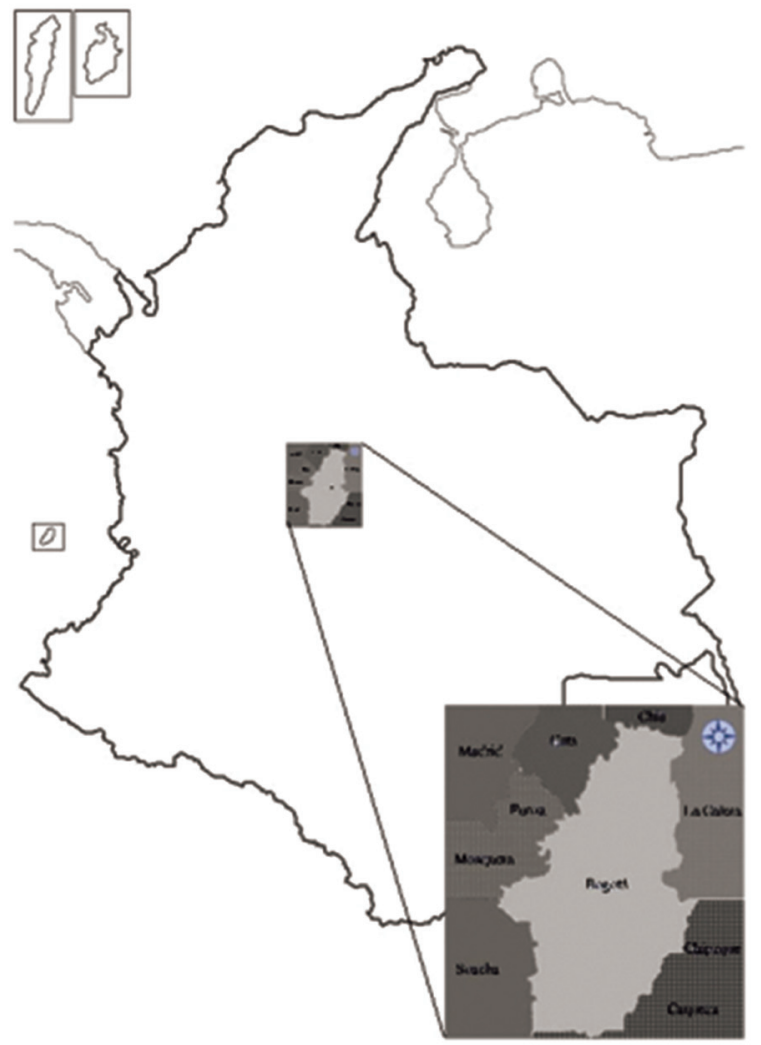

Figura 1. Localización del área de estudio.

\section{Evaluación de la presencia y densidad de insec-} tos de escama en árboles

Los mapas viales de Bogotá y de distribución de árboles fueron superpuestos usando el software ArcView GIS 3.2 - 1999, de forma que se seleccionaron las rutas apropiadas y los sitios de muestreo para cubrir las mayores áreas posibles. Treinta y dos rutas de campo en zonas públicas fueron cubiertas, evaluando: 1) la presencia o ausencia de insectos de escama en cada árbol seleccionado, 2) la severidad y densidad de infestación, por medio de la observación periférica de la proporción de cada árbol cubierto por los insectos, y 3 ) las coordenadas espaciales con GPS. 


\section{Estadística y análisis de datos}

Para evaluar los patrones de la distribución especial de los insectos de escama en Bogotá, se emplearon análisis geoestadísticos que no requirieron representación espacial, tales como el proceso de saturación de Geyer, la función J y el proceso E, los cuales pueden proveer una amplia descripción del sistema. Primero que todo fue necesario determinar si los árboles sanos e infestados estaban mezclados o separados; para esto, cada árbol individual fue clasificado en dos categorías o marcas ("Si" o "No") de acuerdo a la presencia o ausencia de insectos de escama, respectivamente. Para evaluar si los árboles sanos y aquellos con insectos de escama estaban mezclados o separados, se calculó el valor Gamma para el proceso de saturación de Geyer (modelo de interacción de pares, proveniente de un conjunto de datos bivariado - "si, no") (Aryal, 2011). Los valores de Gamma $<1$ describen un patrón "ordenado" o "inhibidor" e indica que los árboles sanos están separados de los enfermos, mientras valores de Gamma > 1 indican un patrón "interactivo", o la presencia de una mezcla de individuos sanos y enfermos (Baddeley, 2010).

Para explorar la primera hipótesis, se realizó una prueba de etiquetado aleatorio para cada especie individual y para el grupo comprendiendo todas las especies en estudio. Esta prueba asigna locaciones como puntos fijos (en este caso árboles) y re-muestrea las marcas (si o no) azarosamente en n simulaciones (50 en este caso), posteriormente compara los resultados con las marcas reales y gráficamente muestra si las categorías corresponden o no a un patrón azaroso para diferentes distancias entre árboles. En este caso, la función $\mathrm{J}$ fue usada para construir la prueba de etiquetado aleatorio (Baddeley, 2010). En el resultado gráfico, el eje-x corresponde a la distancia entre árboles y el eje-y es el resultado de la función $\mathrm{J}\left(\mathrm{Jsi}_{(r)}-\mathrm{J}_{(r)}\right)$, relativa a la presencia de individuos con una marca específica (presencia de plaga "Jsi, ${ }_{(r)}$ ") comparada con aquellos de una marca diferente (ausencia de plaga " $J_{(r)}$ ").

Para confirmar la segunda hipótesis, se usó la prueba de promedio condicional $E(r)$, para elementos marcados propuesta por (Schlather, Ribeiro, \&
Diggle 2004). Esta prueba realiza un diagnóstico de dependencia entre puntos y marcas (en este caso árboles y la densidad de insectos de escama). Esta función compara el promedio de la densidad de insectos de escama con la densidad asignada a un punto al azar (árbol) dado que otro árbol existe a una distancia $r$ del primero (Baddeley, 2010). En este caso, $E(r)$ muestra si la densidad de insectos escama es más alta o baja (curva continua) que el promedio (línea punteada). Dada una distancia r entre árboles, esta línea continua debe ser constante (línea recta) si el punto y la marca son independientes (densidad de insectos de escama independiente de la distancia entre árboles). Esta prueba fue diseñada para procesos estacionarios (espacialmente homogéneos) y una corrección para procesos no estacionarios no ha sido desarrollada. Todos los análisis fueron realizados con el programa Spatstat Ver 1.27-0, para especies individuales y para los datos de todas las especies arbóreas en conjunto.

Finalmente, para visualizar la situación real, la distribución espacial de la severidad de infestación de insectos de escama fue analizada usando el valor porcentual de infestación por árbol individual. Los árboles y sus valores asociados de densidad de insectos de escama fueron proyectados en mapas de Bogotá para cada especie arbórea y para el grupo de todas las especies, usando el software Arc-Map 9.0 (ESRI) - 2006. Esta permite la visualización de la presencia de grupos o áreas con densidades particularmente altos o bajos de insectos de escama.

\section{Resultados}

Los insectos de escama fueron encontrados sobre las cuatro especies seleccionadas en el siguiente orden ascendente: 28 individuos de $C$. bogotensis (21\% de los árboles muestreados), 211 de E. myrtifolia (54\%), 95 de S. molle (61\%) y 140 de F. soatensis (69\%). La densidad de insectos de escama siguió casi el mismo orden de la presencia: $C$. bogotensis $(6.20 \pm 1.60 \%)<$ E. myrtifolia $(12.72 \pm 0.85 \%)$ $<$ F. soatensis $(16.36 \pm 1.69 \%)<$ S. molle $(25.23 \pm$ $2.58 \%)$. Con la excepción de S. molle, todas las especies evaluadas presentaron un valor Gamma $>1$ 
para distancias $\leq 200 \mathrm{~m}$, indicando la presencia de una mezcla de árboles infestados y sanos, sin importar la distancia entre éstos. Este no fue el caso con S. molle, el cual solo presentó una mezcla de arbustos infestados y no infestados a distancias superiores a $5 \mathrm{~m}$.

En la Figura 2, el área gris delimita los intervalos de confianza para datos al azar y la línea continua corresponde a los datos observados para diferentes distancias entre individuos; cuando la línea continua está por dentro del área gris, la ocurrencia simultánea de dos árboles infestados es un evento al azar a la distancia proyectada en el eje-x. La presencia de insectos de escama en los individuos más cercanos (distancias de hasta aproximadamente $40 \mathrm{~m}$ ) no fue azarosa en C. bogotensis (Figura 2A), E. myrtifolia (Figura 2B) y $S$. molle (Figura 2D), mientras las distribución de la presencia de insectos de escama fue al azar en $F$. soatensis y también en el grupo que comprende todas las especies en conjunto (Figura 2E).
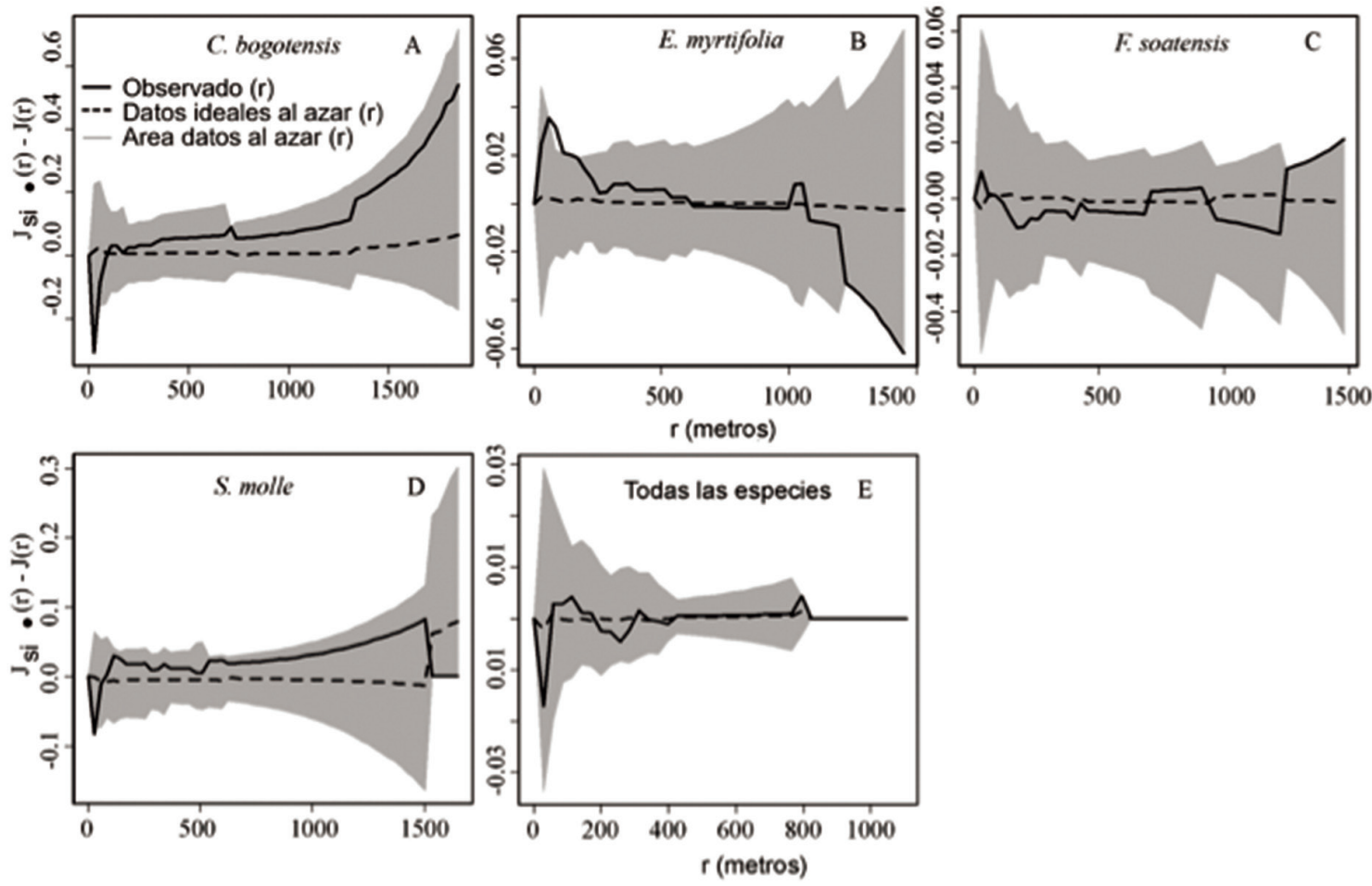

Figura 2. Prueba de etiquetado al azar para la presencia de insectos de escama sobre C. bogotensis (A), E. myrtifolia (B), F. soatensis (C), S. molle (D) y un grupo que comprende todas las especies en conjunto (E).

El eje-x corresponde a las distancias entre árboles y el eje-y explica la presencia de individuos con una marca específica (presencia de plaga) comparada con aquellos con una marca diferente (ausencia de plaga).

La distancia entre árboles afectó la densidad de insectos de escamas de una manera negativa; la proximidad entre árboles contribuye a incrementar la densidad de insectos de escama a diferentes distancias de acuerdo a la especie arbórea, en $C$. bogotensis (prom. 6, incrementó hasta 8\% a distancias de hasta $300 \mathrm{~m}$ Figura 3A), E. myrtifolia (prom. 13, hasta 18\% dentro de 500 m, Figura 3B), S. molle (prom. 25, hasta $33 \%$ dentro de $1900 \mathrm{~m}$, Figura 3D) y en el grupo que comprende todas las especies (prom. 12.2, hasta $16 \%$ dentro de 500 m, Figura 3E); mientras que en los árboles de $F$. soatensis se mantiene la densidad de insectos de escama debajo del promedio a distancias inferiores a $300 \mathrm{~m}$ (hasta un 7\% menos, Figura 3C). 

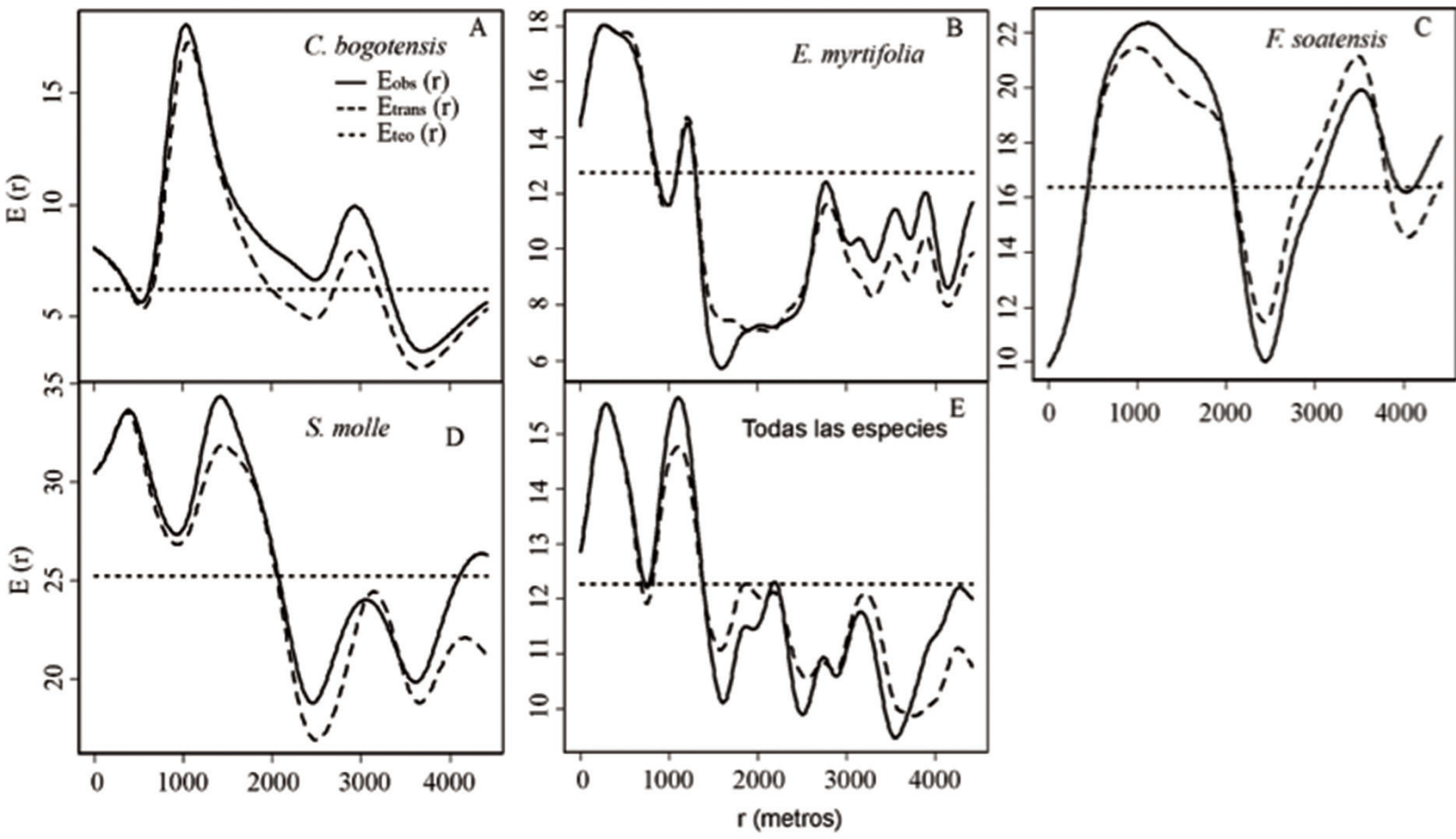

Figura 3. Diagnóstico de dependencia (Emark) entre los puntos (árboles) y las marcas (densidad de insectos de escama) sobre C. bogotensis (A), E. myrtifolia (B), F. soatensis (C), S. molle (D) $y$ el grupo que comprende todas las especies $(E)$, para diferentes distancias entre individuos. El eje-x corresponde a la distancia entre árboles mientras el eje-y $(E(r))$ es el promedio del valor de la marca (densidad de insectos de escama) unido a un punto al azar, donde Eteo corresponde al promedio de todas las marcas, Etrans corresponde a la Emark transformada a cada distancia (r) y Eobs corresponde a la Emark observada para los datos a cada distancia (r).).

Aunque los árboles estén distribuidos por toda la ciudad, las más altas densidades de insectos de escama están en focos que dependen de la especie de árbol hospedero. En C. bogotensis, las mayores densidades de insectos de escama fueron encontradas hacia la zona sudeste de la ciudad (Figura 4A), mientras en $E$. myrtifolia estos estuvieron localizados en la zona noreste y sureste, con bajas severidades de infestación en el centro de la ciudad (Figura 4B). En $F$. soatensis, altas densidades estuvieron distribuidas homogéneamente a lo largo de la ciudad (Figura 4C), mientras en S. molle, cinco grupos de alta densidad fueron observados, cada uno rodeado de arbustos con bajas densidades de insectos de escama (Figura 4D). Finalmente, agrupando todas las especies arbóreas, los individuos con altas y bajas densidades de insectos de escama estuvieron mezclados en toda la ciudad (Figura 4E). 

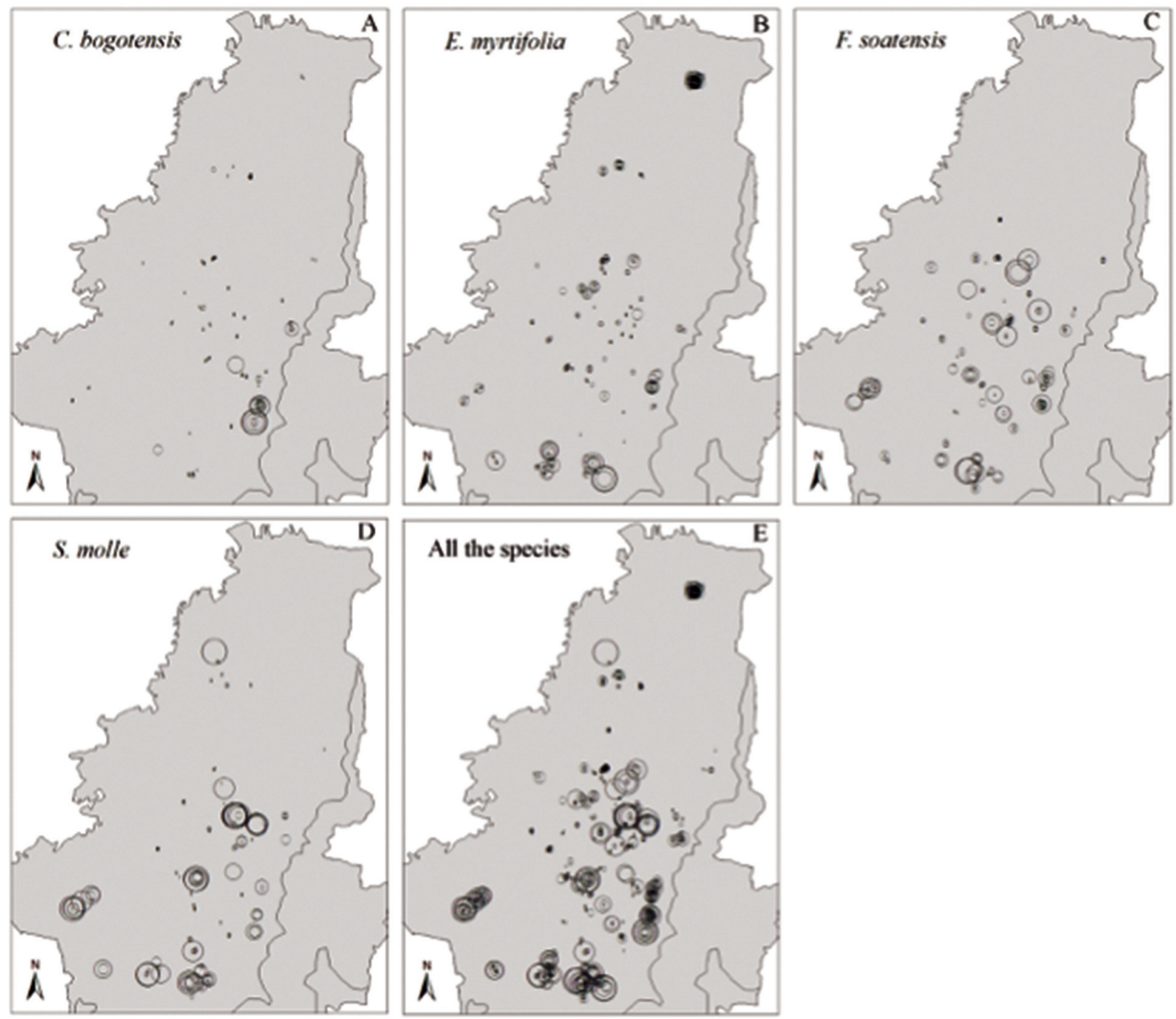

Figura 4. Distribución espacial de la densidad de insectos de escama para C. bogotensis (A), E. myrtifolia (B), $F$. soatensis (C), S. molle (D) y el grupo que comprende todas las especies en conjunto (E) en la zona urbana de Bogotá, Colombia. Cada punto en el esquema corresponde a un círculo, cuyo diámetro es directamente proporcional a la severidad de infestación de insectos de escama.

\section{Discusión}

El uso de diferentes especies arbóreas y arbustivas en la aforestación urbana de Bogotá (Mahecha Vega et al., 2010) recuerda a un policultivo, donde la organización heterogénea de la ciudad, sus parques, avenidas, jardines y áreas disponibles para la siembra de árboles, dictan una distribución poco homogénea en los esquemas de aforestación. Adicionalmente, la alta variabilidad en las condiciones edáficas y ambientales que existen en las ciudades, por ejemplo, patrones de lluvia (Posada \& RamosMontaño, 2012) y pequeñas diferencias locales en temperatura (Meineke et al., 2013), pueden influir en la presencia de insectos de escama.
Lo anterior, en conjunto con la amplia variabilidad genética y rango de edades dentro de cada especie de árboles plantados, asociados con una adaptación local de insectos de escama a hospederos específicos (Hanks \& Denno, 1994) y las relaciones hídricas de las plantas (Hanks \& Denno, 1993), pueden explicar el por qué algunos árboles y arbustos presentan infestación por insectos de escama, mientras otros son sanos a distancias superiores a $5 \mathrm{~m}$. Debido a su pequeño diámetro $E$. myrtifolia y $S$. molle pueden ser sembrados a distancias cortas, pero solo $S$. molle mostró una clara separación entre árboles con o sin insectos de escama a distancias $\leq 5 \mathrm{~m}$; esto puede estar relacionado con la heterogeneidad edáfica encontrada a distancias cortas 
(Jaimes \& Arellano G, 1998; Jin \& Jiang, 2002) y a las relaciones entre las condiciones nutricionales del suelo y la producción de compuestos de defensa (Awmack \& Leather, 2002; Mandzak \& Moore, 1994) por parte de esta especie medicinal.

Solo sobre $F$. soatensis se presentó una distribución azarosa de los insectos (Figura 2C); C. bogotensis, E. myrtifolia y S. molle (Figuras 2A, B y D) fueron afectados principalmente en áreas de hasta $40 \mathrm{~m}$. Esto puede estar relacionado a la densidad de insectos de escama, dado que árboles de la misma especie vegetal, E. myrtifolia, S. molle (Figuras $3 B$ y $D$, Figuras $4 B$ y $D$ ) y en una menor medida, C. bogotensis (Figuras $3 \mathrm{~A}$ y $4 \mathrm{~A}$ ) presentaron densidades de insectos de escama superiores al promedio a distancias $\leq 40 \mathrm{~m}$. Este resultado indica que los insectos de escama tienen una tendencia a afectar, y tener las mayores densidades en árboles cercanos de S. molle $>$ E. myrtifolia $>C$. bogotensis. Esta observación concuerda con los patrones de dispersión encontrados para insectos de escama a partir de centros de infestación tales como árboles altamente infestados, entre otros hospederos (Hoelscher, 1967; Lampson \& Morse, 1993) y también puede ser el resultado de una palatabilidad reducida en $\mathrm{C}$. bogotensis, posiblemente asociada a la presencia de compuestos oleosos (Nuñez-Arévalo, Moreno-Murillo, Quijano-Celis, \& Pino, 2010; Quevedo, Nuñez, \& Moreno Murillo, 2007), tricomas en el género (González Ramírez \& Poveda Álvarez, 2003; Martínez Gordillo \& Espinosa Matias, 2005) o la presencia de látex (Murillo-A, 2004).

La baja especificidad de los insectos de escama, en términos de plantas hospederas y condiciones ambientales (Posada \& Ramos-Montaño, 2012; Raupp, Shrewsbury, \& Herms, 2010; Speight et al., 1998), facilita su amplia dispersión y explica su distribución azarosa sobre F. soatensis (Figura $2 C)$; sin embargo, la densidad de insectos de escama es baja en los árboles de $F$. soatensis más cercanos (Figuras $3 \mathrm{C}$ y $4 \mathrm{C}$ ), sugiriendo un mecanismo de evasión o repulsión entre árboles cercanos. Es posible que este mecanismo pueda estar asociado con una respuesta denso-dependiente (Bohan et al., 2000; Magura, Lövei \& Tóthmérész,
2008; Sotherton, 1985), o puede ser producto de actividades de control antrópicas en respuesta a altas densidades de insectos de escama, ya que F. soatensis es una de las especies arbóreas más abundantes y ampliamente distribuidas en la ciudad, y con altos valores de presencia y densidad de insectos de escama.

Desde un punto de vista global, reuniendo los datos de las cuatro especies arbóreas, la presencia de insectos de escama en la ciudad se ajusta a una distribución azarosa (Figura 2E). Esto concuerda con las observaciones de O'Rourke y Jones (O'Rourke \& Jones, 2011), quienes declaran que la distancia de dispersión no es un factor importante en la regulación de densidades de plagas de insectos altamente dispersivos en paisajes agrícolas. En este caso, sin embargo, la observación detallada en ambientes urbanos indica que la presencia de tales insectos es un fenómeno dependiente de la distancia (no azaroso); los árboles más cercanos (distancias de hasta $40 \mathrm{~m}$ ) de $C$. bogotensis, E. myrtifolia y $S$. molle muestran una presencia de insectos de escama y quizás la transferencia directa entre estas especies.

Adicionalmente, la densidad de insectos de escama tiende a ser más alta a distancias cortas (hasta aproximadamente $800 \mathrm{~m}$, Figura 3E) comparada con árboles más distantes, indicando una relación inversa entre la distancia entre árboles, la incidencia y severidad de insectos de escama de los árboles de las especies indicadas, en donde se cuenta con grandes áreas con densidades de insectos de escama por encima del promedio (Figura 4E) y sugieren la presencia de condiciones tales como zonas calientes (Meineke et al., 2013) o valores de precipitación media (Posada \& Ramos-Montaño, 2012) que son favorables para el desarrollo de altas densidades de insectos de escama (Wang, $\mathrm{Li}, \mathrm{Hu}$, Wu \& Qi, 2009) lo cual se encuentra más allá de los objetivos del presente estudio. Excepciones en distribución de insectos de escama tales como sobre $F$. soatensis sugieren que la presencia de éstos en Bogotá se encuentra al azar (Figura 2C) lo cual hace del tratamiento de plagas, un problema más difícil de resolver. 


\section{Conclusiones}

Los insectos de escama afectan los árboles de Bogotá y están distribuidos en grandes áreas con densidades sobre el promedio; este patrón está definido como un arreglo de pequeños grupos en la mayoría de las especies arbóreas seleccionadas. En insectos de escama, este patrón puede estar asociado con la introducción de hospederos potenciales (árboles) en ambientes urbanos, donde cada especie de árbol o arbusto puede presentar su propia susceptibilidad o a un comportamiento completamente contrastante al patrón general, p. ej. F. soatensis.

El conocimiento de los patrones de distribución de insectos de escama puede contribuir al entendimiento de su dinámica en respuesta a ambientes multi-factoriales que, en conjunto con estudios ambientales específicos y culturales, pueden asistir para el apropiado manejo de esta plaga por parte de los tomadores de decisiones. La distribución de insectos de escama no es azarosa; los focos están localizados cerca de hospederos preferidos, posiblemente en zonas calientes influenciadas con una precipitación promedio favorable que facilita la abundancia de estos insectos.

Dada la amplia distribución de los insectos de escama en la ciudad, es altamente recomendado realizar manejo de plagas de forma directa en áreas con especies arbóreas con altas densidades de plagas, tales como Eugenia myrtifolia, Schinus molle y Croton bogotensis.

\section{Agradecimientos}

El autor agradece a la subdirección científica y técnica del Jardín Botánico de Bogotá, José Celestino Mutis por su apoyo con los mapas y rutas para poder realizar la presente investigación.

\section{Literatuta Citada}

1. Abd-Rabou, S., Badary, H. \& Ahmed, N. (2012). Control measures of two soft scale insects (Hemiptera: Coccidae) infesting guava and mango trees in Egypt. The Journal of Basic \& Applied Zoology, 65(1), 55-61. doi:10.1016/j.jobaz.2012.04.003

2. Agrios, G. N. (2005). Plant pathology (5a Ed.). San Diego: Elsevier Academic Press.

3. Aryal, N. R. (2011). Point Pattern Analysis. The University of York UK.

4. Avila-Olesen, G. A. (2007). Evaluación física y espacial de los principales problemas sanitarios del arbolado del parque metropolitano de Santiago - Chile. Universidad de Chile, Facultad de Ciencias forestales. Recuperado de: http://www.cybertesis.cl/tesis/uchile/2007/ avila_g/sources/avila_g.pdf

5. Awmack, C. S. \& Leather, S. R. (2002). Host plant quality and fecundity in herbivorous insects. Annual Review of Entomology, 47, 817-44. doi:10.1146/annurev. ento.47.091201.145300

6. Baddeley, A. (2010). Analysing spatial point patterns in R. Perth.

7. Becerra, V., González, M., Herrera, M. E. \& Miano, J. L. (2006). Dinámica poblacional de Planococcus ficus Sign. Revista FCA UNCuyo, 38(1), 1-6.

8. Bohan, D. A., Bohan, A. C., Glen, D. M., Symondson, W. O. C., Wiltshire, C. W. \& Hughes, L. (2000). Spatial dynamics of predation by carabid beetles on slugs. Journal of Animal Ecology, 69(3), 367-379. doi:10.1046/j.1365-2656.2000.00399.x

9. Dhingra, O. D. \& Sinclair, J. B. (1995). Basic plant pathology methods (Second edi.). Lewis publishers.

10. Francl, L. J. (2001). The Disease Triangle: A Plant Pathological Paradigm Revisited. The Plant Health Instructor. doi:10.1094/PHI-T-2001-0517-01

11. Fuchs, M., Marsella-Herrick, P., Loeb, G. M., Martinson, T. E. \& Hoch, H. C. (2009). Diversity of ampeloviruses in mealybug and soft scale vectors and in grapevine hosts from leafroll-affected vineyards. Phytopathology, 99(10), 1177-84. doi:10.1094/PHYTO-99-10-1177

12. González Ramírez, J. \& Poveda Álvarez, L. (2003). Dos nuevas especies de Croton (Euphorbiaceae) en el neotrópico. Lankesteriana, 8(3), 7-12.

13. Grantz, D. A., Garner, J. H. B. \& Johnson, D. W. (2003). Ecological effects of particulate matter. Environment International, 29(2-3), 213-39. doi:10.1016/S01604120(02)00181-2

14. Hanks, L. M. \& Denno, R. F. (1993). Natural enemies and plant water relations influence the distribution of an armored scale insect. Ecology, 74(4), 1081-1091.

15. Hanks, L. M., \& Denno, R. F. (1994). Local adaptation in the armored scale insect Pseudaulacapsis pentagona (Homoptera: Diaspididae). Ecology, 75(8), 2301-2310. 
16. Hoelscher, C. E. (1967). Wind dispersal of brown soft scale crawlers, Coccus hesperidum (Homoptera: Coccidae), and Texas citrus mites, Eutetranychus banksi (Acarina: Tetranychidae) from Texas citrus. Annals of the Entomological Society of America, 60(3), 673-678.

17. Hugh, S. \& Cowles, R. (2009). Scale Insect Pests of Connecticut Trees and Ornamentals. University of Connecticut, Integrated Pest Management. Recuperado de: http://www.ct.gov/caes/lib/caes/documents/ publications/fact_sheets/entomology/scale_insect_ pests_of_connecticut_trees_and_ornamentals.pdf

18. Jaimes, E. \& Arellano G, R. (1998). Homogeneidad edáfica relacionada con la biomasa herbácea. Subcuenca baja del rio Castan, Estado Trujillo, Venezuela. Agronomía Tropical, 48(3), 305-333. Recuperado de: http://sian.inia.gob.ve/repositorio/revistas_ci/Agronomia Tropical/at4803/arti/jaimes_e.htm

19. Jin, J. \& Jiang, C. (2002). Spatial variability of soil nutrients and site-specific nutrient management in the P.R. China. Computers and Electronics in Agriculture, 36(23), 165-172. doi:10.1016/S0168-1699(02)00099-6

20. Lampson, L. J. \& Morse, J. G. (1993). Impact of insect growth regulators on black scale, Saissetia oleae (Olivier)(Homoptera:Coccidae) and inter-tree dispersal. Journal of Agricultural Entomology, 9(3), 199-210.

21. Magura, T., Lövei, G. L. \& Tóthmérész, B. (2008). Timeconsistent rearrangement of carabid beetle assemblages by an urbanisation gradient in Hungary. Acta Oecologica, 34(2), 233-243. doi:10.1016/j.actao.2008.05.010.

22. Mahecha Vega, G. E., Sánchez Hurtado, F., Chaparro Guerra, J. A., Cadena Carreño, H. G., Tovar Corzo, G., Villota Ojeda, L. A., ... Quintero, M. A. (2010). Arbolado urbano de Bogotá. Identificación, descripción y bases para su manejo. (Primera ed.). Bogotá - Colombia: Alcaldía Mayor de Bogotá, D.C., Secretaría Distrital de Ambiente, SDA - Jardín Botánico de Bogotá José Celestino Mutis. Recuperado de: http://www.secretariadeambiente.gov.co/sda/libreria/pdf/Arbolado_Urbano_Bogota/Arbolado1.pdf

23. Mandzak, J. M. \& Moore, J. A. (1994). The role of nutrition in the health of inland western forests. Journal of Sustainable Forestry, 2(1-2), 191-210. doi:10.1300/ J091v02n01_09

24. Marcano, R., Nienstaedt, B., Longa, S., \& Malpica, T. (2006). Efecto de la temperatura sobre el tiempo de desarrollo, fecundidad y fertilidad de la cochinilla rosada Maconellicoccus hirsutus (Green), (Hemiptera: Pseudococcidae). Entomotropica, 21(1), 19-22.

25. Martelli, G. P., \& Candresse, T. (2010). Closteroviridae Encyclopedia of Life Sciences. New York: John Wiley \& Sons, Ltd. doi:10.1002/9780470015902.a0000747.pub2.

26. Martínez Gordillo, M. \& Espinosa Matias, S. (2005). Tricomas foliares de Croton sección Barhamia (Euphorbiaceae). Acta Botánica Mexicana, 72, 39-51.

27. Meineke, E. K., Dunn, R. R., Sexton, J. O. \& Frank, S. D. (2013). Urban warming drives insect pest abundance on street trees. PloS One, 8(3), e59687. doi:10.1371/ journal.pone.0059687
28. Murillo-A, J. (2004). Las Euphorbiaceae de Colombia. Biota Colombiana, 5(2), 183-200.

29. Nuñez-Arévalo, L., Moreno-Murillo, B., Quijano-Celis, C. E. \& Pino, J. A. (2010). Composition of the essential oil from leaves of Croton bogotanus Cuatrec Grown in Colombia. Journal of Essential Oil Research, 22(6), 486-487. doi:10.1080/10412905.2010.9700378

30. O'Rourke, M. E. \& Jones, L. E. (2011). Analysis of landscape-scale insect pest dynamics and pesticide use: an empirical and modeling study. Ecological Applications, 21(8), 3199-3210. doi:10.1890/10-1180.1

31. Posada, R. H. \& Forigua Acosta, L. (2008). Eficacia de dos hongos entomopatógenos para el control de Pseudococcus spp. Perez Arbelaezia, 19, 139-149.

32. Posada, R. H. \& Ramos-Montaño, C. (2012). Efecto de la precipitación en la distribución de insectos plaga y síntomas de enfermedades en el arbolado urbano de Bogotá. In Primer congreso latinoamericano de ecología Urbana (p. 14). Buenos Aires - Argentina: Universidad Nacional de General Sarmiento.

33. Quevedo, R., Nuñez, L. \& Moreno Murillo, B. (2007). Contribución al estudio químico y de bioactividad de dos especies nativas: (Croton bogotanus. Cuatr. y Croton funckianus. Cuatr.) Euphorbiaceae. Scientia et Technica Año XIII, 13(33), 391-393.

34. Ramos-Montaño, C. (2012). Flujo vehicular y respuestas eco-fisiológicas del arbolado urbano de Bogotá D.C. In Primer Congreso Latinoamericano de Ecología Urbana (p. 8). Buenos Aires - Argentina: Universidad Nacional de General Sarmiento.

35. Raupp, M. J., Shrewsbury, P. M. \& Herms, D. A. (2010). Ecology of herbivorous arthropods in urban landscapes. Annual Review of Entomology, 55, 19-38. doi:10.1146/annurev-ento-112408-085351

36. Retuerto, R., Fernandez-Lema, B., Rodríguez, R., \& Obeso, J. R. (2004). Increased photosynthetic performance in holly trees infested by scale insects. Functional Ecology, 18, 664-669.

37. Ryan, P. G., Ortmann, H. E. \& Herian, K. (2014). Cascading effects of introduced scale insects on Nesospiza finches at the Tristan da Cunha archipelago. Biological Conservation, 176, 48-53. doi:10.1016/j. biocon.2014.05.020

38. Schlather, M., Ribeiro, P. \& Diggle, P. (2004). Detecting dependence between marks and locations of marked point processes. Journal of the Royal Statistical Society, Series B(66), 79-83.

39. Schmidt, C., Fronza, M., Goettert, M., Geller, F., Luik, S., Flores, E. M., ... Merfort, I. (2009). Biological studies on Brazilian plants used in wound healing. Journal of Ethnopharmacology, 122(3), 523-532. doi:10.1016/j. jep.2009.01.022

40. Sharov, A. A. \& Liebhold, A. M. (1998). Bioeconomics of managing the spread of exotic pest species with barrier zones. Ecological Applications, 8(3), 833-845. doi:10.1890/1051-0761(1998)008[0833:BOMTSO]2.0. $\mathrm{CO} ; 2$. 
41. Sotherton, N. W. (1985). The distribution and abundance of predatory Coleoptera overwintering in field boundaries. Annals of Applied Biology, 106(1), 17-21. doi:10.1111/j.1744-7348.1985.tb03089.x

42. Speight, M. R., Hails, R. S., Gilbert, M. \& Foggo, A. (1998). Horse chesnut scale (Pulvinaria regalis)(Homoptera: Coccidae) and urban host tree environment. Ecology, 79(5), 1503-1513.

43. Vu, N. T., Eastwood, R., Nguyen, C. thi \& Pham, L. Van. (2006). The fig wax scale Ceroplastes rusci (Linnaeus) (Homoptera: Coccidae) in south-east Vietnam: Pest status, life history and biocontrol trials with Eublemma amabilis Moore (Lepidoptera: Noctuidae). Entomological Research, 36(4), 196-201.

44. Vurro, M., Bonciani, B. \& Vannacci, G. (2010). Emerging infectious diseases of crop plants in developing countries: impact on agriculture and socio-economic consequences. Food Security, 2(2), 113-132. doi:10.1007/ s12571-010-0062-7
45. Wang, C., Li, D., Hu, Y., Wu, X. \& Qi, Y. (2009). Research of spatio-temporal analysis of agricultural pest. Proceedings of SPIE, 7492, 74923A-1-74923A-7. doi:10.1117/12.838413

46. Wells, J. D. (1991). Chrysomya megacephala (Diptera: Calliphoridae) has reached the continental United States: review of its biology, pest status, and spread around the world. Journal of Medical Entomology, 28(3), 471-473.

47. Yingping, X., Xianquian, L., Jingping, L. \& Min, T. (1995). The effect of urban air pollution on populations of Eulecanium gigantea (Shinji)(Coccidae) in Taiyuan city, China. Israel Journal of Entomology, 29, 165-168.

48. Zamora, J. E. G., Martínez, N. L., Guerrero, M. A., Fuentes-Guerra, J. M. \& Hernández, C. A. (2015). Superfamilia Coccoidea. Open Course Ware. Sanidad Vegetal. Recuperado de: http://ocwus.us.es/produccion-vegetal/sanidad-vegetal/Sanidad_vegetal/Tema 10_HTML/page_16.htm.
Conflicto de Intereses Los autores declaran no tener ningún conflicto de intereses

Recibido: 13 de octubre de 2015 Aceptado: 03 de noviembre de 2015 